\title{
Emittance growth from luminosity burn-off in future hadron colliders
}

\author{
R. Tomás, J. Keintzel, and S. Papadopoulou \\ CERN, Geneva 23, Switzerland
}

(Received 5 December 2019; accepted 3 March 2020; published 10 March 2020)

\begin{abstract}
Future hadron colliders aim at producing larger integrated luminosities by burning-off an increased fraction of the bunch particles. This burn-off removes particles unevenly in the bunch distribution generating an emittance growth. Analytical equations are derived describing the bunch distribution suffering luminosity burn-off and cooling from synchrotron radiation damping, far from the equilibrium emittance, and possibly including transverse collision offsets. This effect is evaluated for HL-LHC [G. Apollinari et al., Report No. CERN-2017-007-M, 2017], HE-LHC [A. Abada et al., Eur. Phys. J. Spec. Top. 228, 1109 (2019)], and FCC-hh [A. Abada et al., Eur. Phys. J. Spec. Top. 228, 755 (2019).] and included into a step-by-step simulation of the physics fill, together with intrabeam scattering, to evaluate impact on performance.
\end{abstract}

DOI: 10.1103/PhysRevAccelBeams.23.031002

\section{INTRODUCTION AND MOTIVATION}

Understanding the evolution of luminosity during physics fills is of paramount importance in any particle collider in order to optimize its performance. Many efforts have been performed both in the analytical and numerical aspects of luminosity modelling of past and future colliders [1-5]. The effect of emittance growth from luminosity burn-off was already studied in [6,7] for ion collisions in LHC and RHIC with an approximate analytical model and numerical simulations assuming Gaussian distributions throughout the fill. In this paper we derive analytical equations describing the non-Gaussian bunch density distribution in the two transverse dimensions after luminosity burn-off, including synchrotron radiation damping (far from equilibrium emittance) and possible offset collisions. Analytical equations cannot be derived for the case including a crossing angle. Nevertheless, all future hadron colliders consider the use of crab cavities for the full or partial cancellation of crossing angles.

LHC luminosity due to burn-off is illustrated in Fig. 1 for the operational years 2017 and 2018, showing that in LHC a moderate burn-off of about $15 \%$ is typical. Indeed emittance growth from burn-off in LHC has a relatively small impact on performance and it is not considered in this work. The LHC luminosity model is currently being investigated in [8-10].

\footnotetext{
*rogelio.tomas@cern.ch
}

Published by the American Physical Society under the terms of the Creative Commons Attribution 4.0 International license. Further distribution of this work must maintain attribution to the author(s) and the published article's title, journal citation, and DOI.
The emittance growth from burn-off is important for future hadron colliders where it is expected to burn-off up to $80 \%$ of the beam, for the hadron-hadron Future Circular Collider (FCC-hh) [11] case. For the High Luminosity Large Hadron Collider (HL-LHC) [12] and the High Energy LHC (HE-LHC) [13], respectively, about 60\% and $70 \%$ burn-off is expected. Key parameters of these three accelerators are given in Table I. In Fig. 2 simulated instantaneous luminosity, bunch population and rms transverse emittances for nominal physics fills, including synchrotron radiation damping and emittance growth due to intrabeam scattering, are shown for HL-LHC, HE-LHC,

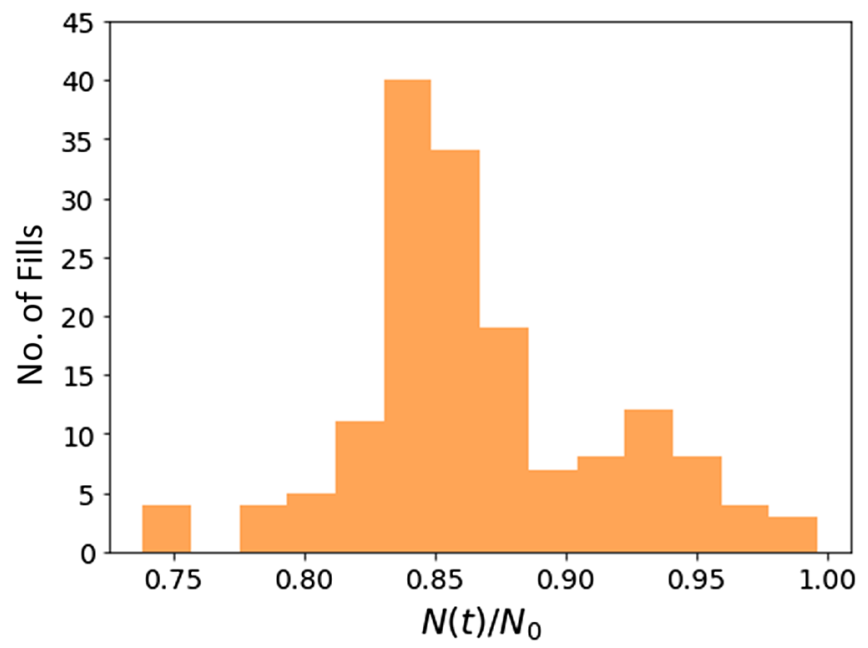

FIG. 1. Histogram of relative final bunch charge considering only luminosity burn-off in 2017 and 2018 LHC fills ending with an operational dump. The final bunch charge is computed from the luminosity signals to avoid including losses not related to luminosity. 
TABLE I. Key parameters for (HL-)LHC, HE-LHC and FCChh for proton operation.

\begin{tabular}{lccc}
\hline \hline Parameter & (HL-)LHC & HE-LHC & FCC-hh \\
\hline c.o.m. energy $[\mathrm{TeV}]$ & 14 & 27 & 100 \\
ppb $\left[10^{11}\right]$ & $(2.2) 1.15$ & 2.2 & 1.0 \\
$\epsilon_{x, y, 0}[\mu \mathrm{m}]$ & $(2.5) 3.75$ & 2.5 & 2.2 \\
$\beta_{x, y}^{*}[\mathrm{~m}]$ & $(0.15) 0.55$ & 0.45 & 0.3 \\
$\sigma_{z}[\mathrm{~mm}]$ & $(75.5) 75.5$ & 90 & $\approx 80$ \\
$\epsilon_{x, y}$ damp. time $[\mathrm{h}]$ & 25.8 & 3.6 & 1.1 \\
Hor. IBS growth time $[\mathrm{h}]$ & 14.7 & 23.5 & 406.7 \\
Inelastic cross section [mb] & 85 & 91 & 108 \\
Total cross section $[\mathrm{mb}]$ & 111 & 126 & 153 \\
Turn-around time $[\mathrm{h}]$ & 2.42 & 3 & 4 \\
$\mathcal{L}_{\text {max }}\left[10^{34} \mathrm{~cm}^{-2} \mathrm{~s}^{-1}\right]$ & $(5$, lev. $) 1$ & 16 & 30 \\
$\mathrm{~L}_{\text {int }}\left[\mathrm{fb} \mathrm{y}^{-1}\right]$ & $(1.9) 0.4$ & 4.5 & 8.0 \\
\hline \hline
\end{tabular}

and FCC-hh. These simulations have been performed with the LEVELLING code [14] already used in [15] to characterize the HL-LHC performance and in [16] for HE-LHC.

Intrabeam scattering also contributes to emittance growth and cannot be neglected in future colliders. However, it will be added here as a perturbation after considering synchrotron radiation damping and emittance growth from luminosity burn-off. In this study we neglect the possible effects from dynamic aperture [17], beam scraping [18], and beam excitations from power converter noise, flux jumps, or crab cavity noise [15,19-21]. To evaluate the intensity loss from luminosity production the total cross section of the proton is used as a pessimistic approximation. The optimistic bound on the performance

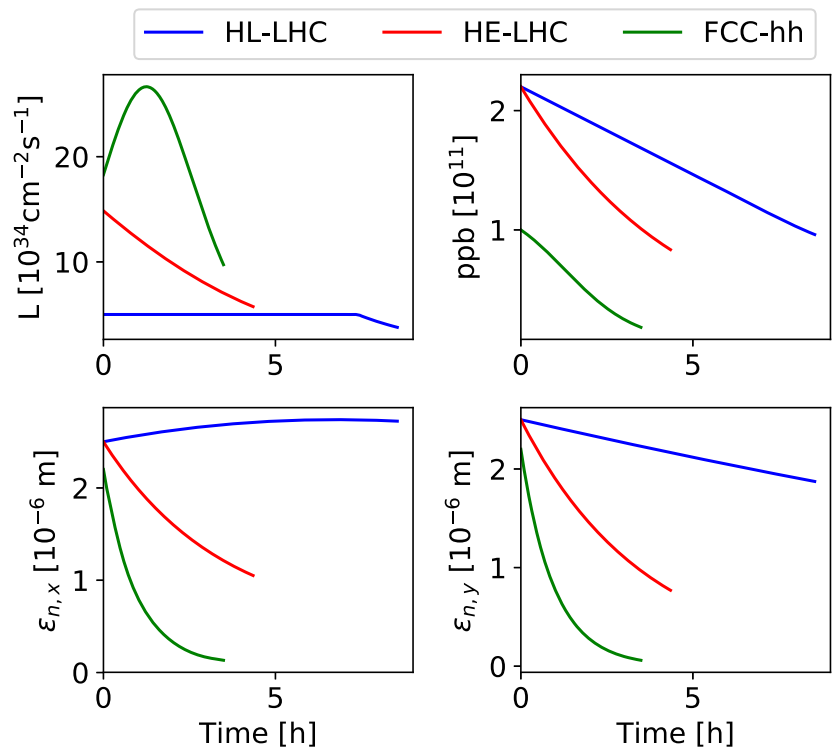

FIG. 2. Simulated luminosity, bunch population and transverse rms emittances for physics fills in HL-LHC, HE-LHC and FCChh including synchrotron radiation damping and intra-beam scattering. loss is also computed by assuming losses generated only from inelastic scatterings. Future high energy hadron colliders will experience significant synchrotron radiation damping but still far from equilibrium emittance. The derivations here do not apply for lepton colliders operating close to the equilibrium emittance.

\section{THEORY}

We assume that the beam distribution is Gaussian at the start of the physics fill in different dimensions,

$$
\rho(z)=\frac{1}{\sigma_{z} \sqrt{2 \pi}} \mathrm{e}^{-\frac{z^{2}}{2 \sigma_{z}^{2}}}
$$

where $z$ can be any of the transverse dimensions $\left\{x, x^{\prime}, y, y^{\prime}\right\}$ and $\sigma_{z}$ is the corresponding beam size. Assuming head-on collisions from two equal bunches the 4-dimensional distribution of particles experiencing collisions is given by [6]

$$
d N\left(x, x^{\prime}, y, y^{\prime}\right)=\sigma_{p} N_{0}^{2} f \rho(x)^{2} \rho(y)^{2} \rho\left(x^{\prime}\right) \rho\left(y^{\prime}\right) \mathrm{d} t \mathrm{~d} v
$$

where $\sigma_{p}$ is the cross section of the proton, $N_{0}$ is the number of particles in the bunches, $f$ is the frequency of collisions and $\mathrm{d} v$ is the 4-dimensional phase-space differential $\mathrm{d} x \mathrm{~d} x^{\prime} \mathrm{d} y \mathrm{~d} y^{\prime}$. The hourglass effect is neglected here as all future colliders plan to operate with $\beta^{*}$ functions larger than the bunch length $\left(\sigma_{z}\right)$, see Table I. Changing to the action-angle variables,

$$
\begin{aligned}
& d N\left(J_{x}, \phi_{x}, J_{y}, \phi_{y}\right) \\
& \quad=\frac{\sigma_{p} N_{0}^{2} f}{\sigma_{x}^{2} \sigma_{y}^{2} \sigma_{x^{\prime}} \sigma_{y^{\prime}} 8 \pi^{3}} \mathrm{e}^{-\left[J_{x}\left(1+\cos ^{2} \phi_{x}\right)+J_{y}\left(1+\cos ^{2} \phi_{y}\right)\right] / \epsilon} \mathrm{d} t \mathrm{~d} v,
\end{aligned}
$$

where $\epsilon$ is the rms geometric beam emittance and $\mathrm{d} v$ now is $\mathrm{d} J_{x} \mathrm{~d} \phi_{x} \mathrm{~d} J_{y} \mathrm{~d} \phi_{y}$. We have assumed equal horizontal and vertical emittances for simplicity. Using $\cos ^{2} \phi=$ $(1+\cos (2 \phi)) / 2$ we obtain

$$
\begin{aligned}
d N & \left(J_{x}, \phi_{x}, J_{y}, \phi_{y}\right) \\
& =\frac{\sigma_{p} N_{0}^{2} f}{\sigma_{x}^{2} \sigma_{y}^{2} \sigma_{x^{\prime}} \sigma_{y^{\prime}} 8 \pi^{3}} \mathrm{e}^{-\left\{J_{x}\left[3+\cos \left(2 \phi_{x}\right)\right]+J_{y}\left[3+\cos \left(2 \phi_{y}\right)\right]\right\} /(2 \epsilon)} \mathrm{d} t \mathrm{~d} v
\end{aligned}
$$

and integrating over the $\phi_{x, y}$ variables, as done in [6], we obtain

$$
\begin{aligned}
& \frac{1}{4 \pi^{2}} \int_{0}^{2 \pi} \int_{0}^{2 \pi} \mathrm{e}^{-\left[J_{x} \cos \left(2 \phi_{x}\right)+J_{y} \cos \left(2 \phi_{y}\right)\right] /(2 \epsilon)} \mathrm{d} \phi_{x} \mathrm{~d} \phi_{y} \\
& \quad=\mathrm{I}_{0}\left(\frac{J_{x}}{2 \epsilon}\right) \mathrm{I}_{0}\left(\frac{J_{y}}{2 \epsilon}\right)
\end{aligned}
$$

where the definition of the modified Bessel function of the first kind has been used, 


$$
\mathrm{I}_{0}(z)=\frac{1}{\pi} \int_{0}^{\pi} \mathrm{e}^{z \cos \phi} \mathrm{d} \phi .
$$

The action distribution of the colliding particles is given by

$$
\begin{aligned}
& d N\left(J_{x}, J_{y}, t=0\right) \\
& =\frac{\sigma_{p} N_{0}^{2} f}{\sigma_{x}^{2} \sigma_{y}^{2} \sigma_{x^{\prime}} \sigma_{y^{\prime}} 2 \pi} \\
& \quad \times \mathrm{e}^{-3\left(J_{x}+J_{y}\right) /(2 \epsilon)} \mathrm{I}_{0}\left(\frac{J_{x}}{2 \epsilon}\right) \mathrm{I}_{0}\left(\frac{J_{y}}{2 \epsilon}\right) \mathrm{d} t \mathrm{~d} J_{x} \mathrm{~d} J_{y},
\end{aligned}
$$

which is the equivalent result as in [6]. We take here a further step by analytically integrating the above equation over time, realizing that $N\left(J_{x}, J_{y}, 0\right)$ is given by

$$
N\left(J_{x}, J_{y}, 0\right)=\frac{N_{0}}{\epsilon^{2}} \mathrm{e}^{-\left(J_{x}+J_{y}\right) / \epsilon},
$$

and incorporating it in Eq. (6) at $t=0$,

$$
\begin{aligned}
d N & \left(J_{x}, J_{y}, 0\right) \\
= & -\frac{\sigma_{p} f N_{0} \mathrm{~d} J_{x} \mathrm{~d} J_{y}}{\epsilon \sqrt{\beta_{x}^{*} \beta_{y}^{*}} 2 \pi} N\left(J_{x}, J_{y}, 0\right) \\
& \times \mathrm{e}^{-\left(J_{x}+J_{y}\right) /(2 \epsilon)} \mathrm{I}_{0}\left(\frac{J_{x}}{2 \epsilon}\right) \mathrm{I}_{0}\left(\frac{J_{y}}{2 \epsilon}\right) \mathrm{d} t .
\end{aligned}
$$

Here we make the ansatz that the same differential equation holds for $N\left(J_{x}, J_{y}, t\right)$ at any $t>0$,

$$
\begin{aligned}
& d N\left(J_{x}, J_{y}, t\right) \\
& =-\frac{\sigma_{p} f N_{0} \mathrm{~d} J_{x} \mathrm{~d} J_{y}}{\epsilon \sqrt{\beta_{x}^{*} \beta_{y}^{*}} 2 \pi} N\left(J_{x}, J_{y}, t\right) \\
& \quad \times \mathrm{e}^{-\left(J_{x}+J_{y}\right) /(2 \epsilon)} \mathrm{I}_{0}\left(\frac{J_{x}}{2 \epsilon}\right) \mathrm{I}_{0}\left(\frac{J_{y}}{2 \epsilon}\right) \mathrm{d} t .
\end{aligned}
$$

It is important to note that no assumption on the shape of $N\left(J_{x}, J_{y}, t\right)$ is taken here and that $\epsilon$ remains constant. Dividing by $N\left(J_{x}, J_{y}, t\right)$ and integrating over $t$ gives

$$
\begin{aligned}
& \ln N\left(J_{x}, J_{y}, t\right)-\ln N\left(J_{x}, J_{y}, 0\right) \\
& =\frac{\sigma_{p} f N_{0}}{\epsilon \sqrt{\beta_{x}^{*} \beta_{y}^{*} 2 \pi}} \mathrm{e}^{-\left(J_{x}+J_{y}\right) /(2 \epsilon)} \mathrm{I}_{0}\left(\frac{J_{x}}{2 \epsilon}\right) \mathrm{I}_{0}\left(\frac{J_{y}}{2 \epsilon}\right) t,
\end{aligned}
$$

which, using Eq. (7), results in

$$
\begin{aligned}
& N\left(J_{x}, J_{y}, t\right) \\
& \quad=\frac{N_{0}}{\epsilon^{2}} \mathrm{e}^{-\left(J_{x}+J_{y}\right) / \epsilon-\frac{\sigma_{p} f N_{0}}{\epsilon \sqrt{\beta_{x}^{*} \beta_{y}^{*}} e^{-\pi}} \mathrm{e}^{-\left(J_{x}+J_{y}\right) /(2 \epsilon)} \mathrm{I}_{0}\left(\frac{J_{x}}{2 \varepsilon}\right) \mathrm{I}_{0}\left(\frac{J_{y}}{2 \epsilon}\right) t} .
\end{aligned}
$$

This equation can be simplified by introducing the scaled Bessel function $\hat{\mathrm{I}}_{0}(x)$ and a new time variable $\tau$ as

$$
\begin{aligned}
& \hat{\mathrm{I}}_{0}(x)=\mathrm{e}^{-x} \mathrm{I}_{0}(x), \\
& \tau=\frac{\sigma_{p} f N_{0}}{\epsilon \sqrt{\beta_{x}^{*} \beta_{y}^{*}} 2 \pi} t,
\end{aligned}
$$

giving

$$
N\left(J_{x}, J_{y}, \tau\right)=\frac{N_{0}}{\epsilon^{2}} \mathrm{e}^{-\left(J_{x}+J_{y}\right) / \epsilon-\hat{\mathrm{I}}_{0}\left(\frac{J_{x}}{2 \epsilon}\right) \hat{I}_{0}\left(\frac{J_{y}}{2 \epsilon}\right) \tau} .
$$

$\tau$ can also be expressed in terms of the initial particle collision rate, $R_{0}$, and the initial number of particles in the bunch, $N_{0}$, as $\tau=R_{0} t /\left(2 N_{0}\right)$. Therefore $\tau=1 / 2$ can be interpreted as the time needed to burn all particles at the initial collision rate. From this equation the total number of particles versus time $N(\tau)$ and the average action $\bar{J}_{x}(\tau)$, which is the effective (or rms) emittance, are given by the double integrals over the transverse action variables as

$$
\begin{gathered}
N(\tau)=\iint N\left(J_{x}, J_{y}, \tau\right) \mathrm{d} J_{x} \mathrm{~d} J_{y}, \\
\bar{J}_{x}(\tau)=\frac{1}{N(\tau)} \iint J_{x} N\left(J_{x}, J_{y}, \tau\right) \mathrm{d} J_{x} \mathrm{~d} J_{y} .
\end{gathered}
$$

It is shown later that including synchrotron radiation damping does not change the above results, being enough to use a new time dependent emittance and a new timelike variable.

To verify the validity of the ansatz and the equations above, simulations of a one dimensional bunch in a collider have been performed including a linear one turn map and burn-off from luminosity computed numerically by placing bunch particles in a grid. The $1 \mathrm{D}$ equivalent formula to Eq. (14), is given by

$$
N\left(J_{x}, \tau\right)=\frac{N_{0}}{\epsilon} \mathrm{e}^{-J_{x} / \epsilon-\hat{\mathrm{I}}_{0}\left(\frac{J_{x}}{2 \epsilon}\right) \tau} .
$$

Figure 3 compares this equation to simulation results, showing a good agreement in the relevant range of burnoff for future hadron colliders.

The actual results in 2 dimensions, using Eq. (14), are shown in Fig. 4. The difference in emittance growth between one and two dimensions is minor. In the extreme case of FCC-hh where up to $80 \%$ of the beam might be burned-off about $40 \%$ relative emittance growth would be expected.

The density distribution along the $x$ coordinate is given by

$$
\rho(x, \tau)=\frac{\iint N\left(\frac{x^{2}+x^{\prime 2}}{2 \epsilon}, J_{y}, \tau\right) \mathrm{d} x^{\prime} \mathrm{d} J_{y}}{\iint N\left(J x, J_{y}, \tau\right) \mathrm{d} J_{x} \mathrm{~d} J_{y}},
$$




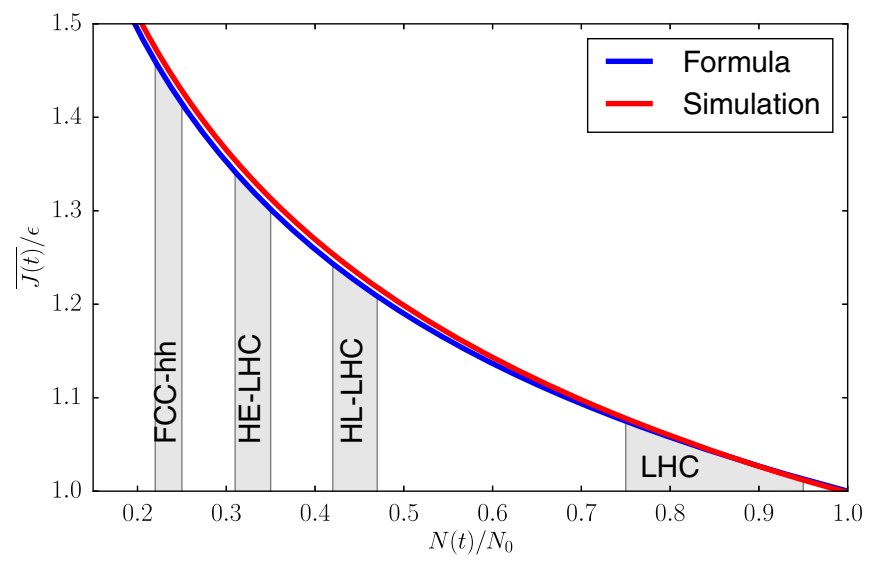

FIG. 3. Relative emittance growth versus remaining bunch intensity from analytical formula in Eq. (17) and simulations considering only one transverse dimension. Shaded regions indicate typical or expected relative intensities after a physics fill.

where, for simplicity, it is evaluated at a location with $\beta_{x}=1 \mathrm{~m}$ and $\alpha_{x}=0 . \rho(x, \tau)$ is illustrated in Fig. 5 for the first four integer values of $\tau$. HL-LHC distribution would be close to the blue curve, while FCC-hh shape would be in between the blue and the yellow distributions.

Expanding Eq. (17) up to first order in $\tau$ gives

$$
N\left(J_{x}, \tau\right)=\frac{N_{0}}{\epsilon} \mathrm{e}^{-J_{x} / \epsilon}\left[1-\tau \hat{\mathrm{I}}_{0}\left(\frac{J_{x}}{2 \epsilon}\right)\right]+O\left(\tau^{2}\right)
$$

and using the following integrals,

$$
\begin{gathered}
\int_{0}^{\infty} \mathrm{e}^{-3 J_{x} /(2 \epsilon)} \mathrm{I}_{0}\left(\frac{J_{x}}{2 \epsilon}\right) \mathrm{d} J_{x}=\frac{\epsilon}{\sqrt{2}}, \\
\int_{0}^{\infty} J_{x} \mathrm{e}^{-3 J_{x} /(2 \epsilon)} \mathrm{I}_{0}\left(\frac{J_{x}}{2 \epsilon}\right) \mathrm{d} J_{x}=\frac{3 \epsilon}{4 \sqrt{2}},
\end{gathered}
$$

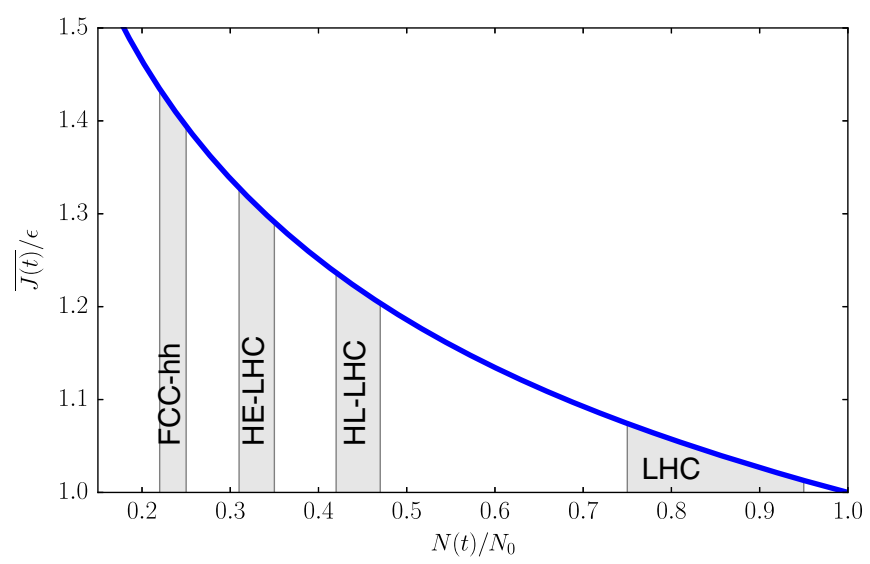

FIG. 4. Relative emittance growth versus remaining bunch intensity from analytical formula considering two transverse dimensions. In case synchrotron radiation damping is considered the $\epsilon$ in the vertical label means $\epsilon(t)$.

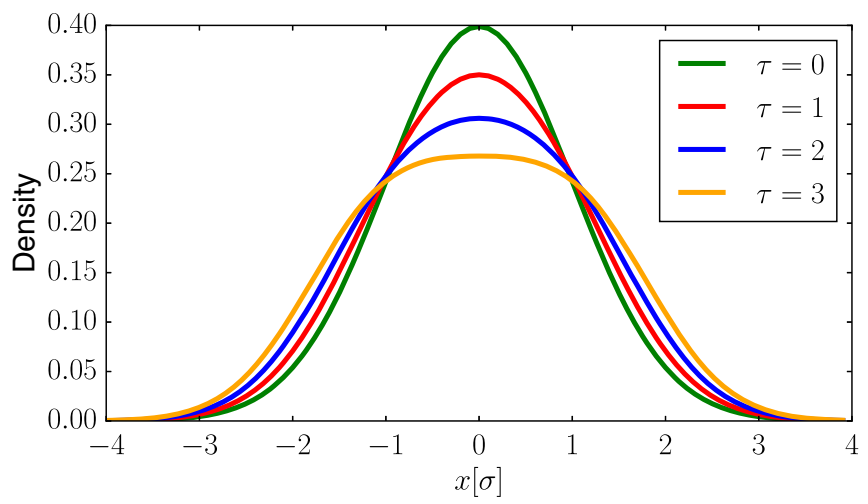

FIG. 5. Particle densities in the horizontal direction for $\tau=0,1$, 2 , and 3 , corresponding to $0 \%, 50 \%, 74 \%$, and $86 \%$ burn-off.

it is easy to relate the average action and the number of particles remaining in the bunch as

$$
\frac{\overline{J(\tau)}}{\epsilon}=\frac{5-N(\tau) / N(0)}{4}+O\left(\tau^{2}\right) .
$$

This relation is illustrated in Fig. 6 .

In hadron colliders beam emittances are much larger than equilibrium emittances and therefore synchrotron radiation damping can be introduced as a shrinking of the action variable as a function of time, typically following an exponential evolution. This can be done in Eq. (9) by using $\epsilon=\epsilon(t)$ and changing the integration action variables to the normalized ones $J_{x, y}^{\prime}=J_{x, y} / \epsilon(t)$

$$
\begin{aligned}
d N\left(J_{x}^{\prime}, J_{y}^{\prime}, t\right)= & -\frac{\sigma_{p} f N_{0}}{\epsilon(t) \sqrt{\beta_{x}^{*} \beta_{y}^{*} 2 \pi}} N\left(J_{x}^{\prime}, J_{y}^{\prime}, t\right) \\
& \times \mathrm{e}^{-\left(J_{x}^{\prime}+J_{y}^{\prime}\right) / 2} \mathrm{I}_{0}\left(\frac{J_{x}^{\prime}}{2}\right) \mathrm{I}_{0}\left(\frac{J_{y}^{\prime}}{2}\right) \mathrm{d} t .
\end{aligned}
$$

Following the same integration steps as above the integration over time needs to include the $\epsilon(t)$ function, which can be grouped into a new timelike variable $\tau$ as

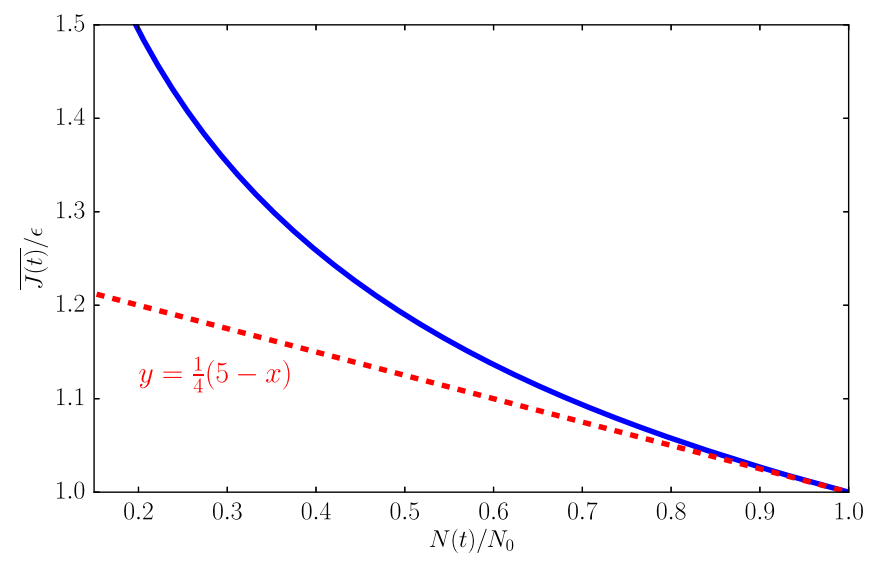

FIG. 6. Emittance growth versus bunch population showing linear approximation from Eq. (22). 


$$
\tau^{\prime}=\frac{\sigma_{p} f N_{0}}{\sqrt{\beta_{x}^{*} \beta_{y}^{*} 2 \pi}} \int_{0}^{t} \frac{\mathrm{d} t}{\epsilon(t)} .
$$

This results in the following equation for the particle density versus $\tau^{\prime}$,

$$
N\left(J_{x}^{\prime}, J_{y}^{\prime}, \tau^{\prime}\right)=N_{0} \mathrm{e}^{-\left(J_{x}^{\prime}+J_{y}^{\prime}\right)-\hat{\mathrm{I}}_{0}\left(\frac{J_{x}^{\prime}}{2}\right) \hat{\mathrm{I}}_{0}\left(\frac{J_{y}^{\prime}}{2}\right) \tau^{\prime}},
$$

which is the same function as in Eq. (14). This implies that the relative emittance growth versus burn-off has the same behavior with or without synchrotron radiation. The only difference being the faster burn-off included in the new variable $\tau^{\prime}$. Figures 4, 5 and 6 are therefore also valid when considering synchrotron radiation damping by replacing $\epsilon$ with $\epsilon(t)$.

Offset collisions might be needed to reduce the luminosity provided to the detector. This luminosity leveling technique is routinely applied in the two low luminosity detectors in the LHC and it has also been experimentally demonstrated in the two high luminosity LHC detectors [22]. Appendix derives analytical equations for the case considering offsets collisions. The main conclusion is that a $1 \sigma$ offset reduces the emittance blow-up from luminosity burn-off by about a factor of 2 .

\section{PERFORMANCE IMPACT ON FUTURE COLLIDERS}

Performance reduction due to emittance growth resulting from particle burn-off is estimated for HL-LHC, HE-LHC, and FCC-hh using the LEVELLING code [14] already used for HL-LHC [15] and HE-LHC [16]. Here we approximate the transverse distributions of the bunch to be Gaussian during the entire physics fill. The emittance is used to reflect the growth from the analytical estimates. Beam parameters are updated step-wise every 3 minutes. Emittance growth due to particle burn-off results from inelastic cross sections. In a real machine, however, the bunch population depends on the total cross-sections, determining pile-up and hence luminosity. Crab cavity rf curvature and the hour-glass effect are included in this evaluation. Total fill time $\left(t_{\text {fill }}\right)$ is optimized to maximize luminosity for each scenario. Evaluating the performance impact based on total cross section burn-off leads to more pessimistic results, compared to scenarios including only inelastic losses. Integrated luminosity reduction is therefore given for these two extreme scenarios, to mark the boundaries of losses observed in a real machine.

The emittance is determined at each step by three growth times, resulting from synchrotron radiation $\left(\tau_{\mathrm{SR}}\right)$, intrabeam scattering $\left(\tau_{\mathrm{IBS}}\right)$, calculated by MAD-X in each step, and emittance growth through burn-off $\left(\tau_{\mathrm{BO}}\right)$ as

$$
\frac{d \epsilon}{d t}=\epsilon\left(-\frac{1}{\tau_{\mathrm{SR}}}+\frac{1}{\tau_{\mathrm{IBS}}}+\frac{1}{\tau_{\mathrm{BO}}}\right) .
$$

$\tau_{\mathrm{BO}}$ is evaluated, based on fills including only synchrotron radiation and burn-off resulting from total cross-sections, leading to an expected emittance growth at the end of the fill of about $20 \%, 30 \%$, and $40 \%$, respectively, for HLLHC, HE-LHC, and FCC-hh, as seen in Fig. 3. Based on fill lengths $\left(t_{\text {fill }}\right)$, including effects of total cross sections, synchrotron radiation damping and predicted emittance growths, $\tau_{\mathrm{BO}}$ is evaluated by

$$
\epsilon_{\text {final }}=\epsilon_{0} \times \mathrm{e}^{\frac{t_{\text {fill }}}{\tau_{\text {BO }}}},
$$

and results in $50.6 \mathrm{~h}, 16.8 \mathrm{~h}$, and $6.0 \mathrm{~h}$, respectively, for HLLHC, HE-LHC, and FCC-hh. Intra-beam scattering is then
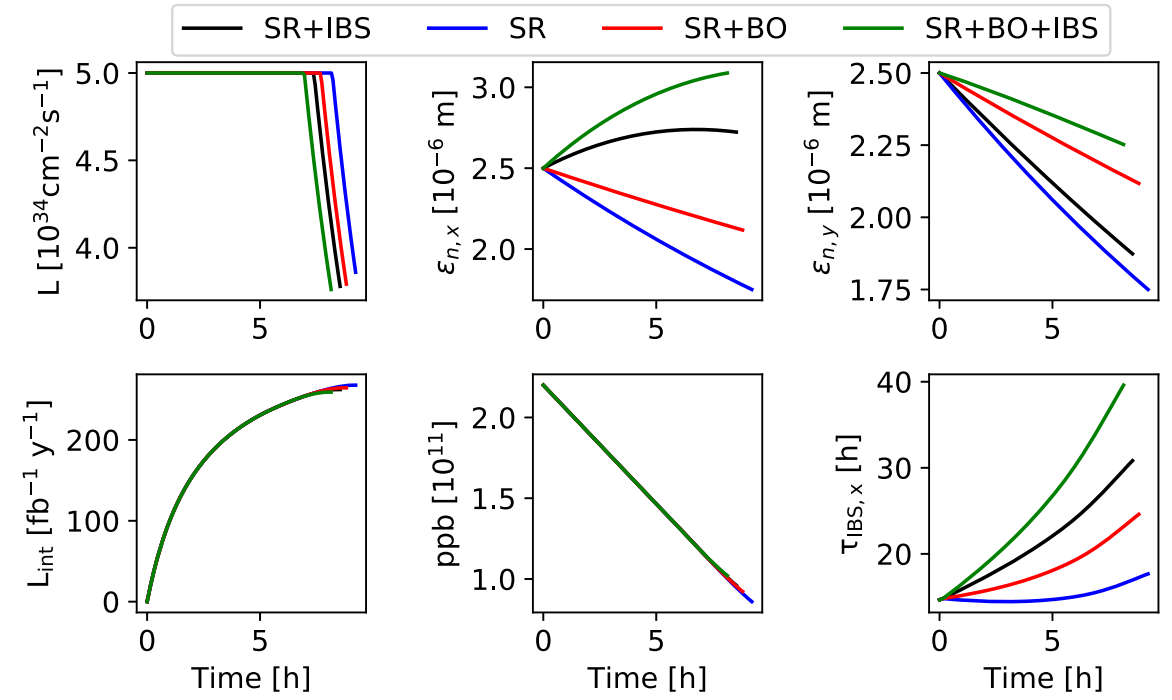

FIG. 7. Simulated physics for HL-LHC, including synchrotron radiation (SR), intrabeam scattering (IBS) or emittance growth due to burn-off (BO). 

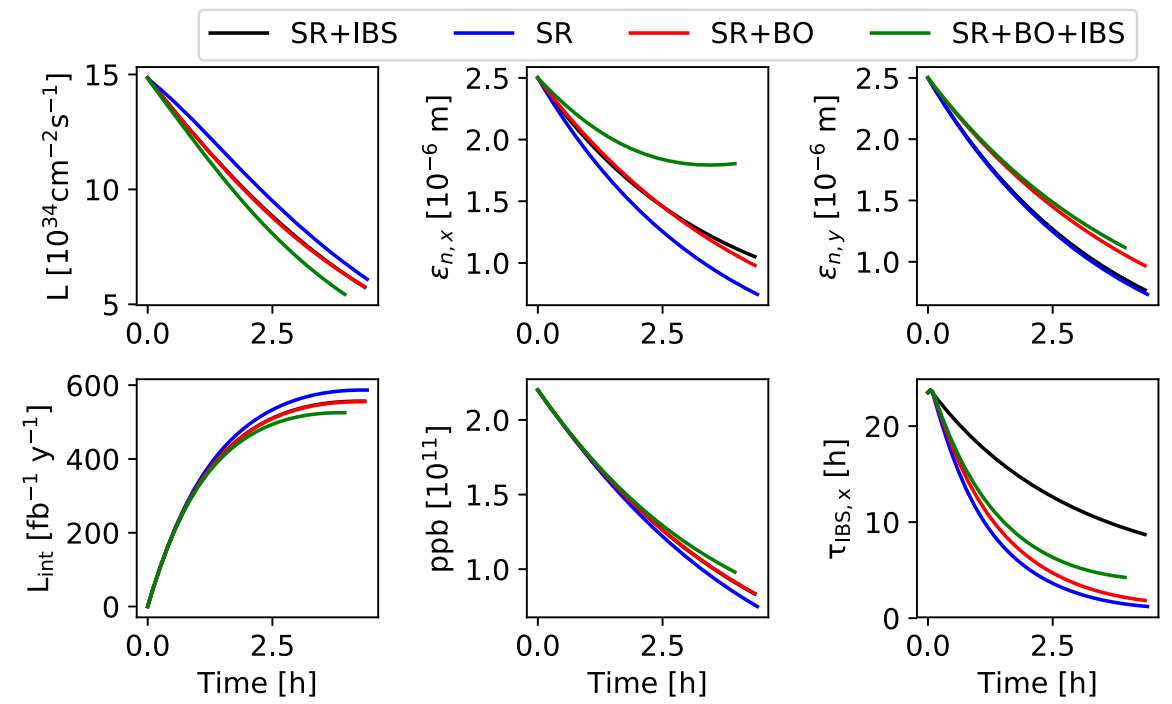

FIG. 8. Simulated physics for HE-LHC, including synchrotron radiation (SR), intrabeam scattering (IBS) or emittance growth due to burn-off (BO).

added as a perturbation to simulate more realistic physics fills. Assuming burn-off only due to inelastic scattering, growth times increase to $66.1 \mathrm{~h}, 20.4 \mathrm{~h}$, and $8.5 \mathrm{~h}$, respectively for HL-LHC, HE-LHC, and FCC-hh. The longitudinal bunch length is kept constant in all scenarios, assuming longitudinal emittance blow-up. Beam parameters used for simulations are taken from Table I.

HL-LHC baseline physics fill, shown in Fig. 7, take losses resulting from the total cross section into account. It features a $\beta^{*}$ leveling technique, with a minimum of $15 \mathrm{~cm}$, and crab-cavities compensating $77 \%$ of a full crossing angle of $500 \mu \mathrm{rad}$ at a center-of-mass energy of $14 \mathrm{TeV}$. In fills including synchrotron radiation and intrabeam scattering, horizontal emittance is already blown up due to strong intrabeam scattering. Burn-off enhances this blow-up by additionally $20 \%$. The effect of synchrotron radiation damping is too small to compensate for arising emittance growth. Moreover, intrabeam scattering is weaker if a burnoff growth times is set simultaneously as $\tau_{\mathrm{IBS}}$ increases. As a result of emittance blow-up due to burn-off assuming total cross-section losses, integrated luminosity is decreased by $1.2 \%$. In the more optimistic scenario assuming intensity loss coming only from inelastic scattering, integrated luminosity is decreased by $0.8 \%$.

For HE-LHC, with a center-of-mass energy of $27 \mathrm{TeV}$, crab cavities compensate for the full crossing angle. In terms of luminosity, physics fills including synchrotron radiation and intrabeam scattering or including synchrotron
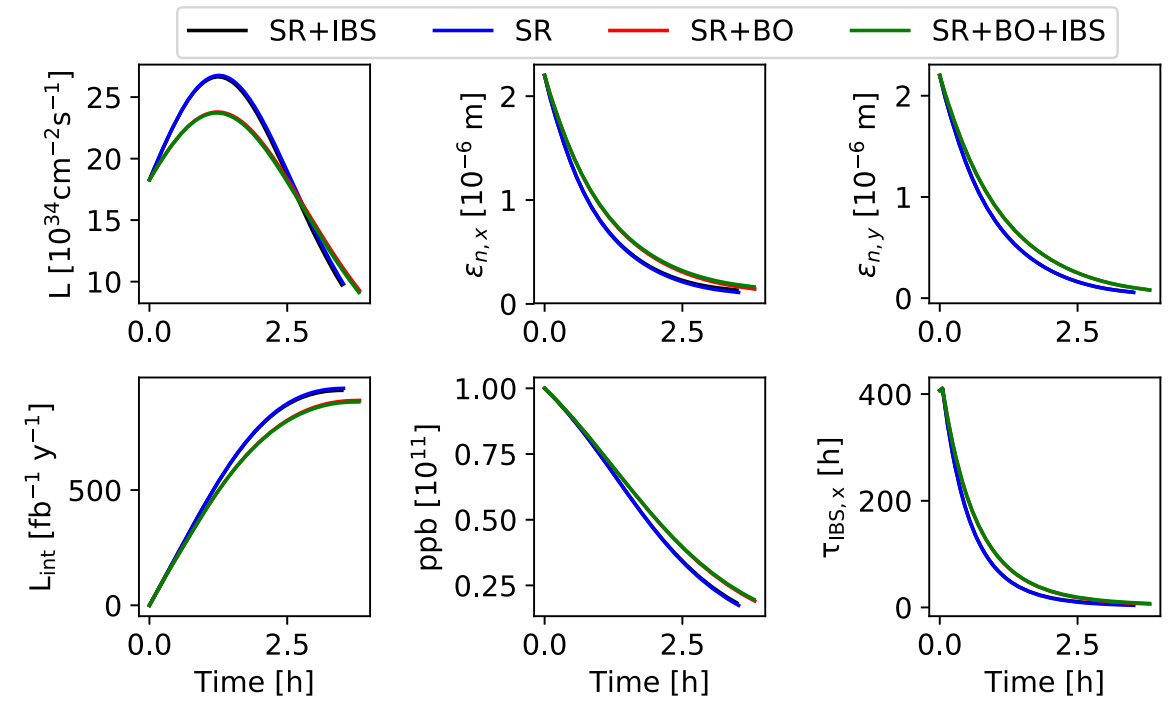

FIG. 9. Simulated physics for FCC-hh, including synchrotron radiation (SR), intrabeam scattering (IBS) or emittance growth due to burn-off (BO). 
TABLE II. Parameters at the end of a physics fill for HL-LHC, HE-LHC, and FCC-hh, where in one case only the synchrotron radiation damping (SR) and intrabeam scattering growth (IBS) are respected. In the other case emittance growth resulting from burn-off (BO) is added additionally.

\begin{tabular}{|c|c|c|c|c|c|c|}
\hline \multirow[b]{2}{*}{ Parameter } & \multicolumn{2}{|c|}{ HL-LHC } & \multicolumn{2}{|c|}{ HE-LHC } & \multicolumn{2}{|c|}{ FCC-hh } \\
\hline & $\mathrm{SR}+\mathrm{IBS}$ & $+\mathrm{BO}$ & $\mathrm{SR}+\mathrm{IBS}$ & $+\mathrm{BO}$ & $\mathrm{SR}+\mathrm{IBS}$ & $+\mathrm{BO}$ \\
\hline$\epsilon_{n, x}[\mu \mathrm{m}]$ & 2.7 & 3.1 & 1.1 & 1.8 & 0.13 & 0.16 \\
\hline$\epsilon_{n, y}[\mu \mathrm{m}]$ & 1.9 & 2.3 & 0.8 & 1.1 & 0.06 & 0.08 \\
\hline$t_{\text {fill }}[\mathrm{h}]$ & 8.5 & 8.1 & 4.4 & 4.0 & 3.5 & 3.8 \\
\hline$\tau_{\mathrm{BO}}[\mathrm{h}]$ & 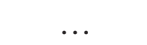 & 50.6 & $\ldots$ & 16.8 & $\ldots$ & 6.0 \\
\hline $\mathrm{ppb}\left[10^{11}\right]$ & 0.96 & 1.0 & 0.8 & 0.98 & 0.18 & 0.20 \\
\hline $\mathrm{L}_{\text {int }}\left[\mathrm{fb} \mathrm{y}^{-1}\right]$ & 262 & 259 & 557 & 525 & 933 & 883 \\
\hline$\Delta L_{\text {int }}[\%]$ & $\ldots$ & -1.2 & $\ldots$ & -6.1 & $\ldots$ & -5.7 \\
\hline
\end{tabular}

radiation and emittance growth due to burn-off from total cross-section, lead to comparable luminosities as shown in Fig. 8. Taking both growth contributions into account gives a horizontal emittance growth of about $64 \%$, with respect to the reference physics fill (synchrotron radiation with intrabeam scattering). One explanation for the severe normalized horizontal emittance increase is the interplay of the two sources, namely intrabeam scattering and growth resulting from particle burn-off. The loss of integrated luminosity results in about $6.1 \%$, assuming burn-off due to total cross section. Assuming only the contribution of inelastic scattering, integrated luminosity is decreased by $4.7 \%$.

For FCC-hh, with a center-of-mass energy of $100 \mathrm{TeV}$, crab cavities compensate for the full crossing angle. Due to the high energy the effect of intrabeam scattering is negligible. The additional emittance growth due to burn-off, however, decreases the integrated luminosity by about 5.7\%, using total cross section losses. Moreover, the maximum instantaneous luminosity is decreased from $26 \times 10^{34} \mathrm{~cm}^{-2} \mathrm{~s}^{-1}$ to $23 \times 10^{34} \mathrm{~cm}^{-2} \mathrm{~s}^{-1}$. Assuming burn-off resulting only from inelastic scattering, integrated luminosity is decreased by $3.7 \%$, where the peak instantaneous luminosity is about $25 \times 10^{34} \mathrm{~cm}^{-2} \mathrm{~s}^{-1}$. FCC-hh fills are shown in Fig. 9, where burn-off from the total cross section are assumed.

Summaries for all three discussed accelerators are given in Table II.

\section{SUMMARY AND CONCLUSIONS}

Analytical equations for emittance growth from luminosity burn-off are derived for the first time. Future hadron colliders aim at unprecedented luminosities by burning up to $80 \%$ of the initial bunch population, as in the case of FCC-hh. For HL-LHC and HE-LHC, respectively, 60\% and $70 \%$ of the bunch particles are expected to be burned-off. The resulting emittance growth due to inelastic particle burn-off is up to $40 \%$ in case of FCC-hh, including synchrotron radiation damping. The resulting particle density $N\left(J_{x}, J_{y}, \tau\right)$ can be expressed as

$$
N\left(J_{x}, J_{y}, \tau^{\prime}\right)=N_{0} \mathrm{e}^{-\left(J_{x}+J_{y}\right)-\hat{\mathrm{I}}_{0}\left(\frac{J_{x}}{2}\right) \hat{\mathrm{I}}_{0}\left(\frac{J_{y}}{2}\right) \tau^{\prime}},
$$

with the timelike variable $\tau^{\prime}$ introduced in Eq. (24). An analytical similar equation is also obtained for collisions with transverse offsets. A one sigma offset reduces the emittance growth resulting from particle burn-off by a factor 2. For machines where luminosity requires to be leveled, like HL-LHC, leveling techniques based on offset collision can mitigate burn-off effects. However, stability considerations may limit the size of the applicable offsets.

Luminosity reduction from burn-off is estimated using a step-by-step simulation of beam parameters during the physics fill, including an emittance growth due to particle burn-off of 20\%, 30\%, and 40\%, respectively for HL-LHC, HE-LHC and FCC-hh. Pessimistic assumptions, taking into account burn-off resulting from total cross sections, the integrated luminosities are found to decrease by about $6 \%$ both for HE-LHC and FCC-hh. For HL-LHC, loss of integrated luminosity due to particle burn-off emittance growth is significantly lower, to about $1.2 \%$.

\section{ACKNOWLEDGMENTS}

We are thankful to G. Arduini, R. Bruce, G. Sterbini and F. Zimmermann for fruitful discussions and comments on the manuscript.

\section{APPENDIX: COLLISIONS WITH AN OFFSET}

When bunches collide with an offset the transverse luminous region is enlarged and it would be expected that the emittance growth due to burn-off is reduced as compared to centered collisions. The distribution of colliding particles with a horizontal offset of $d$ is given by

$d N\left(x, x^{\prime}, y, y^{\prime}\right)=\sigma_{p} N^{2} f \rho(x) \rho(x-d) \rho(y)^{2} \rho\left(y^{\prime}\right)$.

Moving to action-phase variables and following similar steps as above we obtain

$$
\begin{aligned}
& d N\left(J_{x}, \phi_{x}, J_{y}, \phi_{y}\right) \\
& =\frac{\sigma_{p} N^{2} t \mathrm{e}^{-d^{2} /\left(2 \sigma_{x}^{2}\right)}}{\sigma_{x}^{2} \sigma_{y}^{2} \sigma_{x^{\prime}} \sigma_{y^{\prime}} 8 \pi^{3}} \mathrm{~d} t \mathrm{~d} v \\
& \quad \times \mathrm{e}^{-J_{x}\left(3+\cos 2 \phi_{x}\right) /(2 \epsilon)-\sqrt{2 J \beta} d \cos \phi_{x} / \sigma^{2}-J_{y}\left(3+\cos 2 \phi_{y}\right) /(2 \epsilon)} .
\end{aligned}
$$

To integrate over the horizontal phase variable $\phi_{x}$ the following expansions are needed: 


$$
\begin{aligned}
\mathrm{e}^{z \cos \phi} & =\mathrm{I}_{0}(z)+2 \sum_{n=1}^{\infty} \mathrm{I}_{n}(z) \cos n \phi, \\
\frac{1}{2 \pi} \int_{0}^{2 \pi} \mathrm{e}^{a \cos 2 \phi+b \cos \phi} d \phi & =\mathrm{I}_{0}(a) \mathrm{I}_{0}(b)+2 \sum_{n=1}^{\infty} \mathrm{I}_{n}(a) \mathrm{I}_{2 n}(b) .
\end{aligned}
$$

The action distribution of the colliding particles is given by

$$
\begin{aligned}
d N\left(J_{x}, J_{y}, \tau^{\prime}\right)= & \mathrm{e}^{-3\left(J_{x}+J_{y}\right) /(2 \epsilon)} S\left(\frac{J_{x}}{2 \epsilon}, d\right) \mathrm{I}_{0}\left(\frac{J_{y}}{2 \epsilon}\right) \mathrm{d} \tau^{\prime}, \\
S\left(\frac{J_{x}}{2 \epsilon}, d\right)= & \mathrm{I}_{0}\left(\frac{J_{x}}{2 \epsilon}\right) \mathrm{I}_{0}\left(\frac{d}{\sigma_{x}} \sqrt{\frac{2 J_{x}}{\epsilon}}\right) \\
& +2 \sum_{n=1}^{\infty} \mathrm{I}_{2 n}\left(\frac{d}{\sigma_{x}} \sqrt{\frac{2 J_{x}}{\epsilon}}\right) \mathrm{I}_{n}\left(-\frac{J_{x}}{2 \epsilon}\right) .
\end{aligned}
$$

This equation is integrated using the same ansatz as above, obtaining

$$
\begin{aligned}
N\left(J_{x}, J_{y}, \tau\right) & =\frac{N_{0}}{\epsilon^{2}} \mathrm{e}^{-\left(J_{x}+J_{y}\right) / \epsilon-\hat{S}\left(\frac{J_{x}}{2 \epsilon}, d\right) \hat{\mathrm{I}}_{0}\left(\frac{J_{y}}{2 \epsilon}\right) \tau}, \\
\hat{S}\left(\frac{J_{x}}{2 \epsilon}, d\right) & =\mathrm{e}^{-J_{x} /(2 \epsilon)} S\left(\frac{J_{x}}{2 \epsilon}, d\right) .
\end{aligned}
$$

Figure 10 shows the emittance versus bunch intensity for various offsets. A $0.5 \sigma$ offset decreases the emittance growth by about $10 \%$ while a $1 \sigma$ offset decreases it to $50 \%$. This implies that in scenarios where luminosity needs to be leveled due to detector limitations, as in HL-LHC, it will be more efficient to level with offset collisions to partially mitigate emittance blow-up from burn-off. Nevertheless, due to stability considerations average offset in HL-LHC could be only about $0.5 \sigma[23]$.

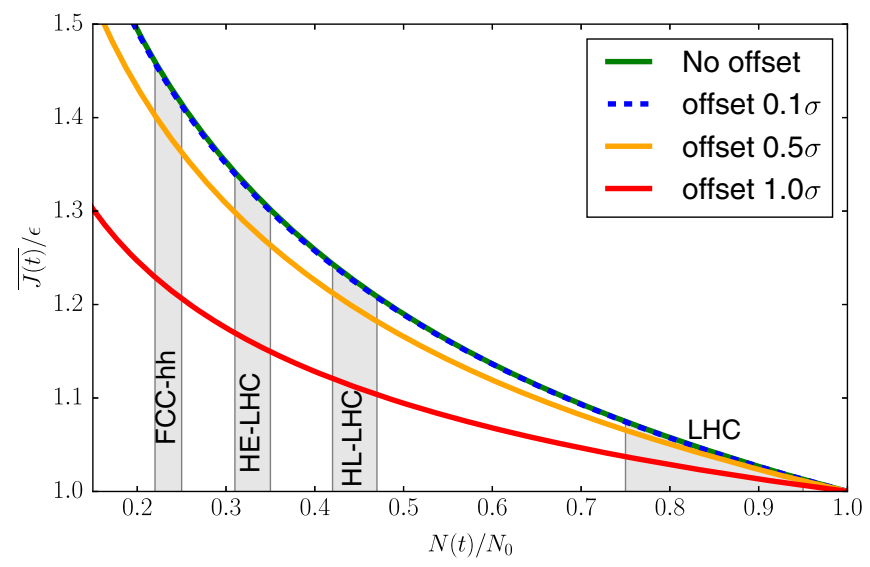

FIG. 10. Emittance growth versus bunch population with offset collisions.
[1] V. Shiltsev and E. McCrory, Characterizing luminosity evolution in the Tevatron, in Proceedings of the 21st Particle Accelerator Conference, Knoxville, TN, 2005 (IEEE, Piscataway, NJ, 2005), p. 2536.

[2] M. J. Syphers, Analytical description of Tevatron integrated luminosity, Report No. FERMILAB-CONF-09154-AD.

[3] C. Gattuso, M. Convery, and M. Syphers, Optimization of integrated luminosity in the Tevatron, Report No. FERMILAB-CONF-09-132-AD.

[4] N. Karastathis, F. Antoniou, I. Efthymiopoulos, M. Hostettler, G. Iadarola, S. Papadopoulou, Y. Papaphilippou, D. Pellegrini, and B. Salvachua, Monitoring and modeling of the LHC luminosity evolution in 2017, in 9th International Particle Accelerator Conference IPAC2018, Vancouver, BC, Canada, pp. 224-227, https://accelconf .web.cern.ch/AccelConf/ipac2018/papers/mopmf052.pdf.

[5] M. Benedikt, D. Schulte, and F. Zimmermann, Optimizing integrated luminosity of future hadron colliders, Phys. Rev. Accel. Beams 18, 101002, 2015.

[6] R. Bruce, Emittance increase caused by core depletion in collisions, arXiv:0911.5627v1.

[7] R. Bruce, J. M. Jowett, M. Blaskiewicz, and W. Fischer, Time evolution of the luminosity of colliding heavy-ion beams in BNL Relativistic Heavy Ion Collider and CERN Large Hadron Collider, Phys. Rev. Accel. Beams 13, 091001 (2010).

[8] F. Antoniou, G. Arduini, Y. Papaphilippou, and G. Papotti, Building a luminosity model for the LHC and HL-LHC, in IPAC 2015, Richmond, USA, TUPTY02, pp. 2042-2045, http://accelconf.web.cern.ch/AccelConf/IPAC2015/papers/ tupty020.pdf.

[9] F. Antoniou, M. Hostettler, G. Iadarola, S. Papadopoulou, Y. Papaphilippou, D. Pellegrini, and G. Trad, Can we predict luminosity, in 7th Evian Workshop, https://indico .cern.ch/event/578001/.

[10] S. Papadopoulou, F. Antoniou, X. Buffat, I. Efthymiopoulos, Y. Papaphilippou, T. Persson, and G. Sterbini, Update on Luminosity model including coupling, noise and burnoff for the emittance growth prediction, 158th HiLumi WP2 Meeting, https://indico.cern.ch/event/844767/.

[11] A. Abada et al., FCC-hh: The Hadron Collider, Eur. Phys. J. Spec. Top. 228, 755 (2019).

[12] G. Apollinari et al., High-Luminosity Large Hadron Collider (HL-LHC): Technical Design Report V. 0.1, Report No. CERN-2017-007-M, 2017.

[13] A. Abada et al., HE-LHC: The High-Energy Large Hadron Collider, Eur. Phys. J. Spec. Top. 228, 1109 (2019).

[14] L. M. Medrano, Levelling code. https://github.com/ lmedinam/Levelling.

[15] L. Medina, R. Tomás, G. Arduini, and M. Napsucialea, Assessment of the performance of High Luminosity LHC operational scenarios: Integrated luminosity and effective pile-up density, Can. J. Phys. 97, 498 (2018).10.1139/cjp2018-0291

[16] J. Keintzel, Optics design and performance aspects of the HE-LHC, Report No. CERN-THESIS-2018-177.

[17] M. Giovannozzi and F. F. Van der Veken, Description of the luminosity evolution for the CERN LHC including 
dynamic aperture effects, Part I: The model, Nucl. Instrum. Methods Phys. Res., Sect. A 905, 171 (2018).

[18] H. Burkhardt and R. Schmidt, Intensity and luminosity after beam scraping, Report No. CERN-AB-2004-032 (ABP), https://cds.cern.ch/record/777311/files/ab-2004-032.pdf.

[19] J. C. de Portugal, R. Tomás, L. Fiscarelli, D. Gamba, and M. Martino, Impact of flux jumps in future colliders, Phys. Rev. Accel. Beams 23, 011001 (2020).

[20] K. Ohmi et al., Response of colliding beam-beam system to harmonic excitation due to crab-cavity rf phase modulation, Phys. Rev. Accel. Beams 14, 111003 (2011).

[21] P. Baudrenghien and T. Mastoridis, Transverse emittance growth due to $\mathrm{rf}$ noise in the high-luminosity LHC crab cavities, Phys. Rev. Accel. Beams 18, 101001 (2015).
[22] X. Buffat, G. Arduini, E. Bravin, G. Iadarola, E. Métral, Y. Papaphilippou, D. Pellegrini, S. Redaelli, B. Salvachua, M. Solfarloli, G. Trad, D. Valuch, J. Wenninger, J. Barranco, T. Pieloni, C. Tambasco, and M. Crouch, Long-range and head-on beam-beam interactions: What are the limits?, in 7th Evian Workshop on LHC beam operation, Evian Les Bains, France (2016), pp. 133-140, http://cds.cern.ch/ record/2293522/.

[23] E. Métral et al., Update of the HL-LHC operational scenarios for proton operation, Report No. CERN-ACCNOTE-2018-0002, https://cds.cern.ch/record/2301292/ files/CERN-ACC-NOTE-2018-0002.pdf. 